\title{
Signaux moléculaires et mécaniques intervenant dans la différenciation des cellules tendineuses
}

\author{
Ludovic Gaut, Marie-Ange Bonnin, Nicolas Robert, Mathias Mericskay, Delphine Duprez
}

Ludovic Gaut

CNRS UMR 7622,

IBPS-Laboratoire

de Biologie

du Développement

F-75005, Paris, France

Inserm U1156, F-75005

Paris, France

Sorbonne Universités,

UPMC Univ Paris 06,

IBPS, F-75005 Paris,

France

CNRS UMR 8256 ,

IBPS-Adaptation

Biologique et

Vieillissement, F-75005,

Paris, France

Inserm U1164, F-75005

Paris, France

Marie-Ange Bonnin

Nicolas Robert

Delphine Duprez

CNRS UMR 7622 ,

IBPS-Laboratoire

de Biologie

du Développement,

F-75005, Paris, France

Inserm U1156, F-75005

Paris, France

Sorbonne Universités,

UPMC Univ Paris 06 ,

IBPS, F-75005 Paris,

France

Mathias Mericskay

Sorbonne Universités,

UPMC Univ Paris 06

IBPS, F-75005 Paris,

France

CNRS UMR 8256

IBPS-Adaptation

Biologique

et Vieillissement

F-75005, Paris, France

Inserm U1164, F-75005

Paris, France
Les tendons sont des formes uniques de tissu conjonctif du système musculo-squelettique. Ils sont formés par une matrice dense de fibres de collagène de type I qui sont orientées parallèlement à l'axe du tendon. Cette organisation spécifique leur donne la capacité de supporter les forces générées par le muscle pour les transmettre à l'os, permettant ainsi le mouvement. Le développement, l'homéostasie et la réparation du tendon reposent sur une combinaison spécifique de facteurs de transcription, de facteurs de croissance ainsi que de paramètres mécaniques régulant la production et l'assemblage des fibres de collagène [1, 2]. Les cascades moléculaires régissant la différenciation du tendon ne sont pas aussi bien décrites que celles des cellules musculaires. Toutefois, l'étude du tendon a été rendue possible grâce à la découverte de certains acteurs moléculaires tels que le facteur de transcription Scx [1-3], le plus spécifique et précoce dans le développement du tendon, ou encore le facteur de transcription mécanosensitif Egr1 [1, 2, 4, 5]. Au cours des dernières années, de nombreuses études ont montré l'importance des forces mécaniques générées par les cellules ou les tissus au cours du développement ou des processus de différenciation cellulaire [6]. Notre objectif est de comprendre comment la cellule de tendon va intégrer les signaux mécaniques et d'identifier quelles voies de signalisation seront activées en aval de ces signaux pour déclencher la différenciation tendineuse.

La mécanobiologie du tendon a été étudiée in vivo au cours du développement. Une paralysie des embryons de poulet au stade E7.5 a été induite par traitement au bromure de décaméthonium (DMB), un agoniste du récepteur à l'acétylcholine bloquant la jonction neuromusculaire [7]. Le traitement au $\mathrm{DMB}$ va induire une paralysie musculaire et donc interrompre les mouvements rythmiques effectués par l'embryon (Figure 1A). Chez les embryons paralysés, l'expression du marqueur de tendon SCX est diminuée dès $5 \mathrm{~h}$ et à $48 \mathrm{~h}$ de traitement (Figure 1A). Ce défaut a notamment été observé avant la diminution des marqueurs musculaires PAX7 et MYF5 (Figure 1A), indiquant que le changement observé est dû à une absence de contraction plutôt qu'à une perte d'un signal biochimique provenant du muscle adjacent.

La mécanobiologie a également été étudiée dans un système in vitro mimant la formation d'un tendon in vitro. Des cultures de cellules souches dans un système en trois dimensions (3D), consistant en un tube de gel de fibrine maintenu sous tension, miment la formation d'un tendon in vitro (Figure 1B) [8]. Des tendons in vitro ont ainsi été réalisés en utilisant une lignée murine de cellules souches mésenchymateuses (CSM), les C3H10T1/2. La comparaison de l'expression de différents gènes dans les CSM cultivées en $3 \mathrm{D}$ versus $2 \mathrm{D}$ met en évidence une augmentation des marqueurs de tendon Scx, et Col1a1 ainsi que du facteur de transcription mécanosensitif Egr1 (Figure 1B). La perte de tension de ces tendons in vitro (après section) conduit à la diminution de l'expression d'Egr1 et des gènes de tendon, Scx et Col1a1 (Figure 1C). L'expression forcée de Egr1 dans les cellules C3H10T1/2 permet d'empêcher la diminution des marqueurs de tendons dans ces tendons in vitro sans tension (Figure 1D). Ces résultats démontrent l'importance des forces mécaniques dans le maintien de l'identité tendon des CSM. De plus, ils montrent l'implication du facteur de transcription Egr1 en aval des forces mécaniques dans le maintien des marqueurs de tendons.

Ces résultats soulignent l'importance des signaux mécaniques pour le développement du tendon et la différenciation tendineuse à partir des cellules souches. Cependant, les liens existant entre les signaux mécaniques et moléculaires restent à être identifiés dans le contexte de la différentiation du tendon. 
A

Embryon de poulet (E7.5)

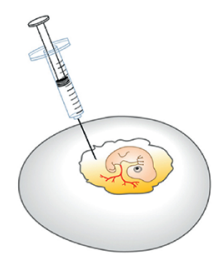

Traitement DMB

(5h or $48 \mathrm{~h})$

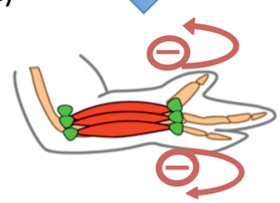

B

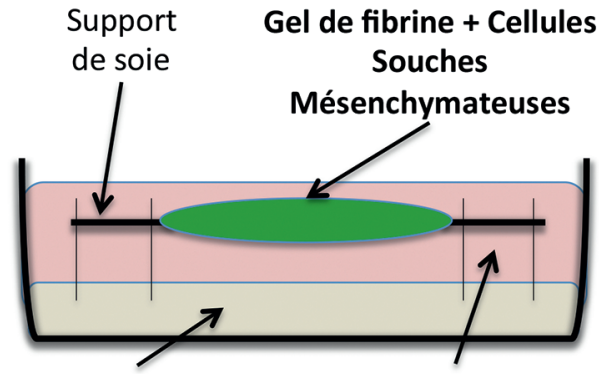

Matrice de silicone

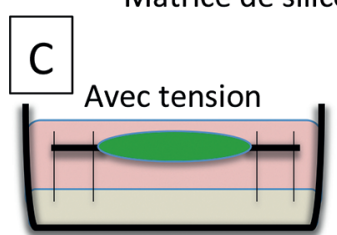

Milieu de culture

Sans tension

\section{Expression des gènes de lignages cellulaires} dans les ailes d'embryons traités au DMB

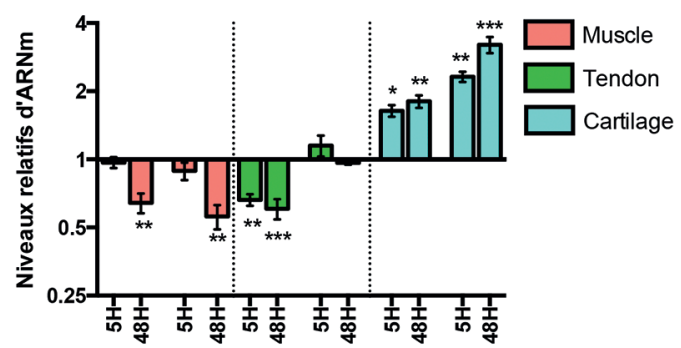

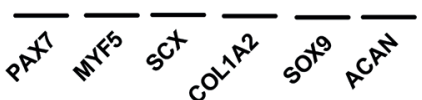

Expression des gènes associés au tendon des cellules en 3D comparée à celle des cultures en 2D

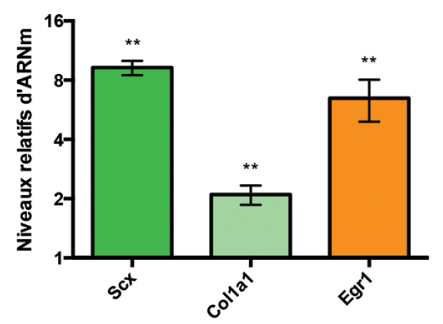

C3H10T1/2 sans tension versus

C3H10T1/2 avec tension

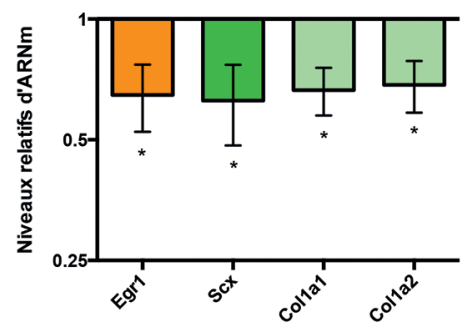

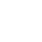
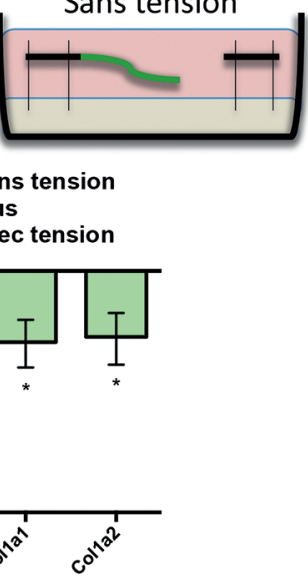

Figure 1

Signaux moléculaires et mécaniques intervenant dans la différenciation des cellules tendineuses.

(A) Procédure expérimentale du traitement au bromure de décaméthonium (DMB) induisant une inhibition des mouvements (flèches rouges) et expression des gènes associés aux différents lignages cellulaires. (B) Représentation d'une construction 3D de tendon in vitro et graphe d'expression des gènes dans des cultures cellulaires 3D comparée aux cultures $2 \mathrm{D}$. (C) Représentation des constructions 3D avec tension (gauche) et sans tension (droit) et graphe d'expression des gènes associés aux tendons dans les 3D sans tension us avec tension. (D) Représentation des constructions 3D surexprimant Egr1 (3D-Egr1) avec tension (gauche) et sans tension (droit) et graphe d'expression des gènes associés aux tendons dans les 3D-Egr1 avec tension us sans tension. Tests statistiques utilisés sont les tests non-paramétriques de Mann-Whitney. ${ }^{*}=\mathrm{p}$-value $\leq 0,05 ;{ }^{* * *}=\mathrm{p}$-value $\leq 0,01 ;{ }^{* * * *}=\mathrm{p}$-value $\leq 0,001$.

\section{Oral communications and Posters Awards 2015}

Molecular and mechanical signals underlying

\section{LIENS D'INTÉRÊT}

Les auteurs déclarent n'avoir aucun lien d'intérêt concernant les données publiées dans cet article. tendon cell differentiation 


\section{RÉFÉRENCES}

1. Gaut L, Duprez D. Tendon development and diseases. Wiley Interdiscip Rev Dev Biol 2016 ; 5 : 5-23.

2. Huang $\mathrm{AH}, \mathrm{Lu} \mathrm{HH}$, Schweitzer R. Molecular regulation of tendon cell fate during development. J Orthop Res 2015 ; 33 : 800-12.

3. Schweitzer R, Chyung JH, Murtaugh LC, et al. Analysis of the tendon cell fate using scleraxis, a specific marker for tendons and ligaments. Development 2001; 128 : 3855-66.

4. Lejard V, Blais F, Guerquin MJ, et al. EGR1 and EGR2 involvement in vertebrate tendon differentiation. $J$ Biol Chem 2011 286 : 5855-67.
5. Guerquin MJ, Charvet B, Nourissat G, et al. Transcription factor EGR1 directs tendon differentiation and promotes tendon repair. J Clin Invest 2013; 123 : 3564-76.

6. Mammoto T, Mammoto A, Ingber DE. Mechanobiology and developmental control. Annu Rev Cell Dev Biol 2013; 29 27-61

7. Nowlan N, Sharpe J, Roddy K, et al. Mechanobiology of embryonic skeletal development: Insights from animal models. Birth Defects Res C Embryo Today 2010 ; 90 : 203-13.

8. Bayer M, Yeung C, Kadler K, et al. The initiation of embryoniclike collagen fibrillogenesis by adult human tendon fibroblasts when cultured under tension. Biomaterials 2010 ; 31 : 4889-97.

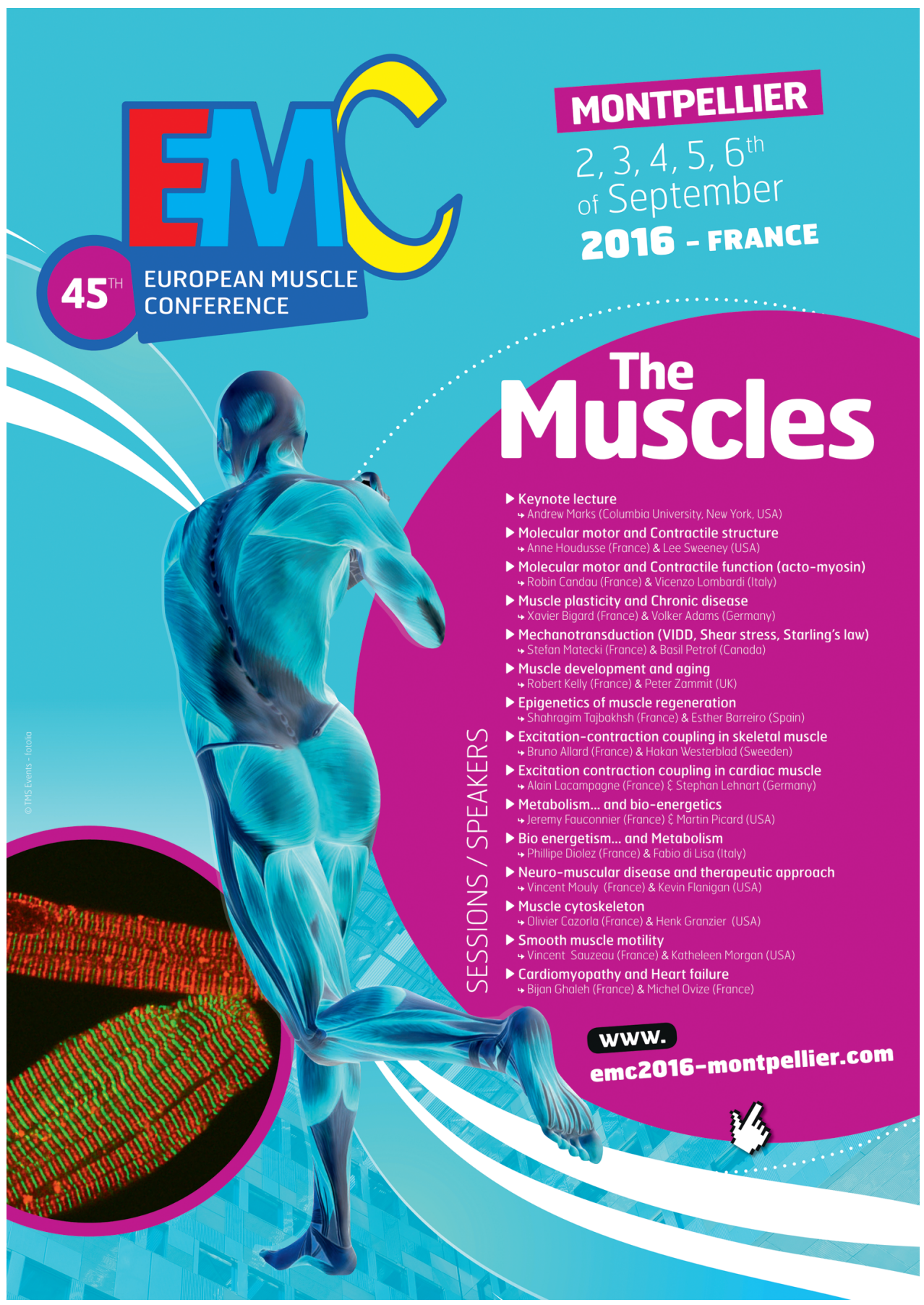

